\title{
Double Fuzzy Point Extension of the Two-step Fuzzy Rule Interpolation Methods
}

\section{Zoltán Krizsán, Szilveszter Kovács}

University of Miskolc

Egyetemváros

3515 Miskolc, Hungary

krizsan@iit.uni-miskolc.hu, szkovacs@iit.uni-miskolc.hu

\begin{abstract}
The "Double Fuzzy Point" rule representation opens a new dimension for expressing changes of fuzziness in fuzzy rule-based systems. In the case of standard "Fuzzy Point" rule representations, it is difficult to describe fuzzy functions in which crisp observations are required to have fuzzy conclusions, or in which an increase in the fuzziness of observations leads to reduced fuzziness in conclusions. These problems are mainly due to a lack of information. A fuzzy point rule determines the connection between a pair of fuzzy sets taken from the domain and the range of the rule. Expressing the fuzzy function through a set of fuzzy points and fuzzy interpolation between pairs of those points, each fuzzy point can be considered as a node point with given location and fuzziness. In common, sparse rule-base definitions, these node points are usually disjunctive on the domain, defining only single antecedent-consequent fuzziness connections at the location of the fuzzy points. However, this kind of information is insufficient when the goal is to express changes in the fuzziness of a given location in the domain. One solution to this problem is the double fuzzy point rule representation concept. Double fuzzy points are pairs of fuzzy points which share the same reference locations, but have different fuzziness properties. The existence of two different fuzziness values in a single location within the domain creates new possibilities for introducing fuzzy interpolation methods capable of interpolating not only between locations, but between changes in local fuzziness values as well. The main goal of this paper is to discuss how two-step Fuzzy Rule Interpolation methods can be adapted to be able to handle the double fuzzy point concept. To this end, an approach referred to as the Generalized Double Fuzzy Point Methodology (GDFPM) is proposed.
\end{abstract}

Keywords: fuzzy rule interpolation; interpolation of the fuzziness; fuzzy function; double fuzzy point rule representation 


\section{Introduction}

There are numerous Fuzzy Rule Interpolation (FRI) methods which have appeared in the literature. One of the first methods was published by Kóczy and Hirota (KH method [1]). The $\mathrm{KH}$ method can only handle convex and normal fuzzy (CNF) sets in single dimensional antecedent universes, determining the conclusion from the $\alpha$-cuts of the two rules which immediately surround the observation. The KH method inspired many subsequent approaches, such as the modified $\alpha$-cut based interpolation (MACI) method [2]. MACI transforms fuzzy sets into vector representations, computes the conclusion based on those representations, and finally transforms the conclusion back to the original space. The first FRI method capable of explicitly dealing with "fuzziness" appeared in the "conservation of relative fuzziness" (CRF) method, which was proposed by Kóczy et al. in [3] for single antecedent dimensions. CRF uses the two closest surrounding rules to the observation. It stipulates that the rate of the left (right) fuzziness of the conclusion and the fuzziness of the rule consequents should be the same as the rate of the right (left) fuzziness of the observation and the fuzziness of the two surrounding rule antecedents. A multidimensional extension of the CRF method, known as IMUL, was proposed in [4] ("An improved fuzzy interpolation technique for multidimensional input spaces"). IMUL is a combination of CRF and the multidimensional MACI methods.

In parallel with these developments, a rather different two-step method was proposed by Bouchon-Meunier et al. [5], [6]. At the first step their "analogy-based interpolation" calculates the reference point of the conclusion. This step is simple interpolation based on the reference point distances of the observation and the rule antecedents. In the second step the FRI method constructs the shape of the conclusion according its similarity (distinguishability) to the rule consequents to be the same as the similarity (distinguishability) of the corresponding rule antecedents and the observation. Another two-step method concept is presented in the "General Methodology" (GM suggested by Baranyi et al. in [7]). GM extends the first step of the original analogy-based interpolation to the generation of interpolated "intermediate rules" in the reference point position of the observation. In the second step, a single rule reasoning method (revision function) is applied to determine the final fuzzy conclusion based on the similarity of the fuzzy observation and an "interpolated" observation. In this way, GM can handle arbitrarily shaped fuzzy sets. An extension of GM appeared in the work of Shen et al. [8]. The suggested "scale and move transformation" extends GM with extrapolation. Practical applications of GM appear in the "Least Squares Method" ("LESFRI"), in the "FRI based on Subsethood Values" ("FRISUV") as well as in the "Polar a Cut" interpolation ("FRIPOC") suggested by Johanyák et al. in [9], [10], [11] and [12]. As a single rule reasoning step FRIPOC calculates the similarity of fuzzy sets based on their polar cuts. 
In the remainder of the paper, the above "two-step" methods will be studied in detail, with the goal of extending them to be able to adopt the "double fuzzy" point rule representation concept. An improvement, referred to as the Generalized Double Fuzzy Point Methodology (GDFPM), is proposed for the case of SISO Mamdani fuzzy systems (i.e., Mamdani systems with one input and one output dimension).

Many of the above mentioned FRI methods and a sparse fuzzy model identification tool are available as open source code MATLAB Toolbox (Johanyák et al. [13], [14], [15]). Systematic model-based fuzzy control approaches are presented in [16].

\section{Definitions and Notations}

This section introduces elementary definitions and concepts and notations utilized in later parts of the paper. Scalar values are denoted by lowercase letters, e.g. $\{a, b, c$, ratio,$\ldots\}$; fuzzy sets are denoted by capital letters, e.g. $\{A, B, P, Q, \ldots\}$; and the letter $R$ is reserved to denote fuzzy rules of the form IF $x=A$, THEN $y=B$, or $R: A \rightarrow B$ for short. The letters $X, Y$, and $S$ are reserved for the input-output universes and for the third dimension of geometrical representations, respectively (see later). $x$ and $y$ are used to denote the observation and the conclusion. Antecedent fuzzy sets are denoted by $A\left(A \in L^{X}\right)$; consequent sets by $B\left(B \in L^{Y}\right)$ where $L^{X}$ and $L^{Y}$ are fuzzy spaces on $X$ and $Y$, respectively.

There are some common concepts which are followed by all of the FRI methods when calculating the similarity between fuzzy sets. Many of the FRI methods define similarity as distances in every important $\alpha$ value $(\alpha=[0 . .1])$ of the $\alpha$ cuts. As this similarity concept requires all of the $\alpha$-cuts to be known in advance, its use is restricted to CNF sets (e.g. LESFRI [9]). Another possible fuzzy set similarity calculation is based on the polar coordinate system and polar cuts. This method has the advantage that it is also suitable for calculating the similarity of subnormal fuzzy sets. The FRIPOC method, introduced by Johanyák in [10], is currently the only FRI method in the literature which uses this technique. The method calculates the consequent for every $\theta$ angle $(0 \leq \theta \leq 180)$ in the domain where the reference point of the fuzzy set (e.g. the centre of the core, or the centre of gravity) is also the reference point of the polar coordinate system. Further methods for calculating the similarity of fuzzy sets include the "scale and move transformation" [8], which is based on the parameters of scaling and translation necessary to transform one fuzzy set into the shape of the other.

It can be stated that at the moment there is no common, universally accepted method used to represent the similarity of two arbitrarily shaped fuzzy sets. In the remainder of the paper, methods applying $\alpha$-cuts and polar cuts for similarity calculations will be studied in more detail. 
Finally, there are some common guidelines followed by all of the FRI methods, which make up the "axiomatic approach of fuzzy interpolation" (an overview can be found in [17], [18] and [19]). Two of these axioms - "avoiding invalid conclusions" and "preserving linearity" - will be studied in this paper. The axiom of "avoiding invalid conclusions" (also referred to as "validity of the mapping" in [19]) stipulates that the conclusion generated by the FRI method should be a valid fuzzy set. The axiom of "preserving linearity" (also referred to as "shape invariance of the mapping" in [19]) stipulates that the conclusion generated by the FRI should have a piecewise linear shape, provided that the observation is based on linguistic terms with piecewise linear shapes.

Definition 1 (fuzziness): Several fuzziness definitions can be found in the literature. An easily interpretable definition was introduced by Kóczy, Hirota and Gedeon in [3] in the following form:

$f_{A, L}=\inf \left\{[A]_{1}\right\}-\inf \left\{[A]_{0+}\right\}$,

$f_{A, U}=\sup \left\{[A]_{0+}\right\}-\sup \left\{[A]_{1}\right\}$,

where $f_{A, L}$ and $f_{A, U}$ are the "lower" and "upper" fuzziness values, $\left[\inf \left\{[A]_{1}\right\}, \sup \left\{[A]_{1}\right\}\right]$ is the core, and $\left[\inf \left\{[A]_{0+}\right\}, \sup \left\{[A]_{0+}\right\}\right]$ is the support of fuzzy set $A$.

In later parts of this paper, the concept of fuzziness will be used in terms of definition 1.

Definition 2 (double fuzzy point rule): A double fuzzy point rule consists of two overlapping fuzzy rules (P,Q) with the same reference points [20].

\section{The Double Fuzzy Point Rule Representation}

A number of Fuzzy Rule Interpolation (FRI) methods exist which use a variety of different computational concepts, but most of them handle changes in fuzziness in similar ways. This is because of the "Monotonicity" condition, which was first defined in [18] (condition "I2") for the single dimensional antecedent case, and was extended in [19] ("Property 6.") to multidimensional antecedents in the following manner: If $f_{A^{*} I}<f_{A^{*} I I}$ in all dimensions (such that $A^{*} I$ is more specific than $\left.A^{*} I I\right)$ then $f_{B^{*} I}<f_{B^{*} I I}$ holds as well. According to the condition, it is not possible to reverse changes of fuzziness in the conclusion. Moreover, a singleton conclusion $f_{B^{*} I}=0$ can be gained only if the observation is a singleton as well (i.e., $f_{A^{*} I}=0$ ).

The "double fuzzy point" rule representation was proposed in [20] in order to extend the classical fuzzy point concept so that changes of fuzziness in fuzzy rules could also be expressed. The "double fuzzy point" rule is an extension of the single fuzzy point rule representation to two overlapping fuzzy rules (cf. 
Definition 1). The fuzzy rule pairs share the same reference points, i.e. in both rules, the corresponding antecedent and consequent fuzzy sets have the same reference points, but apart from this condition, they can have different fuzziness properties. Differences in antecedent fuzziness define the domain, and differences in consequent fuzziness define the range of the fuzziness interpolation [20].

The $\mathrm{i}^{\text {th }}$ rule of the double fuzzy point rule representation has the following form:

$A_{i}^{p, q} \rightarrow B_{i}^{p, q}$,

which combines two overlapping fuzzy rules: $A_{i}^{p} \rightarrow B_{i}^{p}$ and $A_{i}^{q} \rightarrow B_{i}^{q}$, such that both the antecedent and the consequent fuzzy sets in the overlapping rules have the same reference point. Details depend on the way in which reference points are defined, e.g. in triangular linguistic terms the reference point might be the core of the fuzzy set. In this case, the conditions $\operatorname{core}\left(A_{i}^{p}\right)=\operatorname{core}\left(A_{i}^{q}\right), \operatorname{core}\left(B_{i}^{p}\right)=$ $\operatorname{core}\left(B_{i}^{q}\right)$ would hold (see e.g. on Fig. 1 and on Fig. 2).

Based on the above, the double fuzzy point rule-base $R^{p, q}$ can be considered as the superposition of two overlapping rule-bases, $R^{p}$ and $R^{q}$.

According to the double fuzzy point rule representation concept [20], fuzziness interpolation requires an observation $A^{*}$ within the fuzziness domain of the double fuzzy point rule:

$f_{A^{*}} \in\left[f_{A^{q}}, f_{A^{p}}\right]$

and it generates a conclusion $B^{*}$ within the fuzziness range of the double fuzzy point rule:

$f_{B^{*}} \in\left[f_{B^{q}}, f_{B^{p}}\right]$

Depending on the $p, q$ part of the double fuzzy point rule, the direction of change in fuzziness can remain the same, or may be reversed. The direction of change in fuzziness remains the same (see e.g. on Fig. 1) if:

$f_{A^{q}} \leq f_{A^{*}} \leq f_{A^{p}}$ and $f_{B^{q}} \leq f_{B^{*}} \leq f_{B^{p}}$

or

$f_{A^{q}} \geq f_{A^{*}} \geq f_{A^{p}}$ and $f_{B} q \geq f_{B^{*}} \geq f_{B} p$,

The direction of change in fuzziness is reversed (see e.g. on Fig. 2) if:

$f_{A^{q}} \leq f_{A^{*}} \leq f_{A^{p}}$ and $f_{B^{q}} \geq f_{B^{*}} \geq f_{B^{p}}$

or

$f_{A^{q}} \geq f_{A^{*}} \geq f_{A^{p}}$ and, $f_{B} q \leq f_{B^{*}} \leq f_{B} p$. 


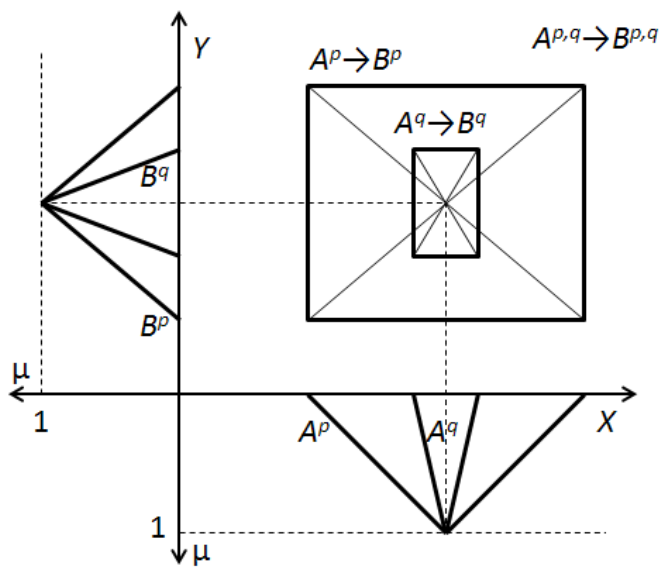

Figure 1

"Double fuzzy point" rule representation when the direction of change in fuzziness remains the same [20]

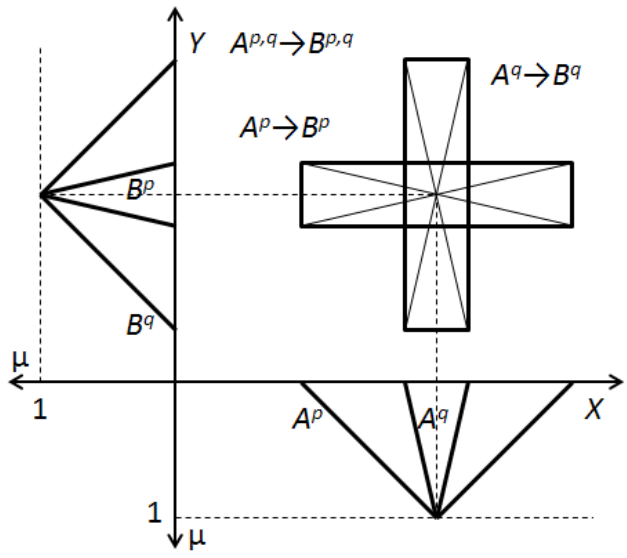

Figure 2

"Double fuzzy point" rule representation when the direction of change in fuzziness is reversed [20].

\section{Double Fuzzy Point Extension of Two Step FRI Methods}

In this section, a novel extension of the two-step FRI method concept (following the generalized methodology [7]) is introduced to support double fuzzy point rule representations. Some additional properties of the newly obtained family of twostep double fuzzy point FRI methods are also examined, such as the validity of the conclusion and the preservation of linearity. 
The proposed "Generalized Double Fuzzy Point Methodology" (GDFPM) can be applied as a guideline for the double fuzzy point adaptation of any two-step FRI method.

GDFPM can be used to extend any two-step FRI method with the ability to handle the $R^{p, q}$ double fuzzy point rule-base, and with a special additional step enabling the interpolation of fuzziness for the final conclusion. As GDFPM is based on two-step FRI methods, the limitations and preconditions of the original two-step FRI method used will be inherited by GDFPM.

The first step of two-step FRI methods is the generation of a temporal interpolated fuzzy rule at the reference point of the observation. In the case of double fuzzy point extended FRI methods (GDFPM), a temporal interpolated fuzzy rule pair is generated, one for each of the two fuzzy rule sets $R^{p}$ and $R^{q}$ in the position of the observation. If the observation is within the fuzziness domain (4) covered by the antecedent fuzzy sets $A_{i}^{p}, A_{i}^{q}$ in every input dimension, then the fuzzy conclusion can be obtained through interpolation. In other cases, the fuzzy conclusion can be considered as an extrapolation of fuzziness. In this paper, extrapolation is not discussed.

The second step of the GDFPM method proposed here is the determination of the conclusion based on the observation $\left(A^{*}\right)$ and the temporal interpolated rule pair $\left(A_{i}^{p} \rightarrow B_{i}^{p}, A_{i}^{q} \rightarrow B_{i}^{q}\right)$ generated in the previous step. The concept of double fuzzy rule representation suggests that the property of "fuzziness similarity ratio preservation" should hold between the triplets $A_{i}^{p}, A^{*}, A_{i}^{q}$ and $B_{i}^{p}, B^{*}, B_{i}^{q}$. Therefore, as a final step of GDFPM, the single rule reasoning step of the original two-step method is replaced with a new "fuzziness similarity ratio preservation reasoning" step.

The "fuzziness similarity ratio preservation reasoning" step is an extension of the common single rule reasoning concept. Rather than preserving the fuzzy similarity of the observation and the rule antecedent to the conclusion and the rule consequent, it preserves the fuzziness similarity ratio of the observation and the two antecedents of the double fuzzy rule to the conclusion and the two corresponding consequents. Generally speaking, the fuzziness similarity ratios must be equal on both the antecedent and the consequent sides:

fuzzinessRatio $\left(A^{p, q}, x\right)=\operatorname{fuzzinessRatio}\left(B^{p, q}, y\right)$.

This "fuzziness similarity ratio" is calculated in the same manner in which fuzzy similarity was calculated in the single rule reasoning step of the original two-step method, but this time it is calculated based on the double fuzzy rule. Therefore, as discussed earlier, the interpretation of the similarity ratio preservation strongly depends on the FRI technique used. In the following, the previously mentioned $\alpha$ cut based and polar cut based fuzzy similarity calculations will be studied in more detail. 
When using $\alpha$-cut based methods (e.g. LESFRI [9], [10]), the similarity ratio can be determined based on the rate of $\alpha$-cut distances $\left(d_{\alpha}\right)$ (see Fig. 3):

fuzzinessRatio $_{L}\left(A^{p, q}, x\right)_{\alpha}=\frac{d_{\alpha}\left(K_{A L}^{p}, K_{x L}\right)}{d_{\alpha}\left(K_{x L}, K_{A L}^{q}\right)}$,
fuzzinessRatio $_{U}\left(A^{p, q}, x\right)_{\alpha}=\frac{d_{\alpha}\left(K_{A U}^{p}, K_{x U}\right)}{d_{\alpha}\left(K_{x U}, K_{A U}^{q}\right)}$,

where " $L$ " denotes the lower, and " $U$ " denotes the upper $\alpha$-cut endpoint distances.

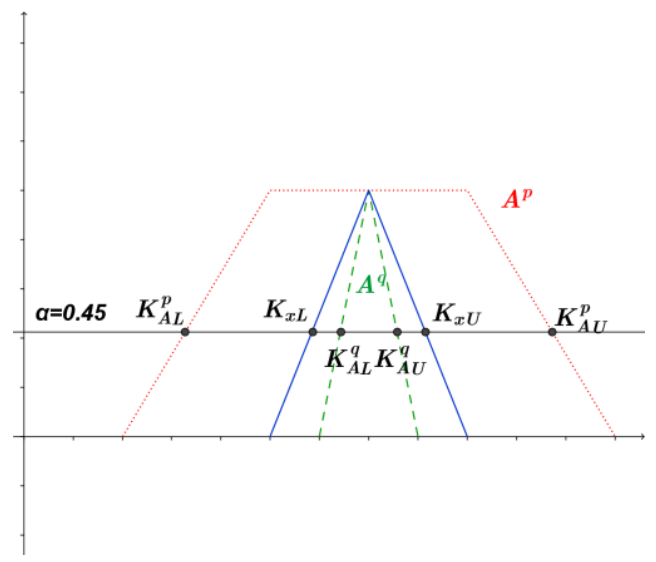

Figure 3

" $\alpha$-cut based" fuzziness similarity ratio

When using polar cut based methods (e.g. FRIPOC [12]), the similarity ratio can be determined based on the rate of polar distances $\left(d_{\theta}\right)$ (see Fig. 4):

fuzzinessRatio $\left(A^{p, q}, x\right)_{\theta}=\frac{d_{\theta}\left(K_{A}^{p}, K_{x}\right)}{d_{\theta}\left(K_{x}, K_{A}^{q}\right)}$

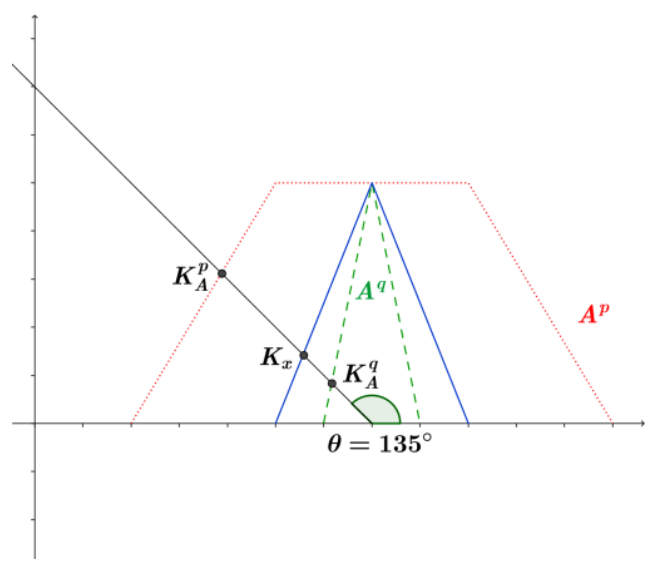

Figure 4

"Polar cut based" fuzziness similarity ratio 
Finally, the conclusion $y$ can be determined based on requirement of equality between the antecedent and consequent side fuzziness similarity ratios. In the case of $\alpha$-cut based methods (see e.g. on Fig. 5 and Fig. 6):

$\forall \alpha \in(0,1]:$

fuzzinessRatio $\left(B^{p, q}, y\right)_{\alpha}=\operatorname{fuzzinessRatio}\left(A^{p, q}, x\right)_{\alpha}$,

$\frac{d_{\alpha}\left(K_{B L}^{p}, K_{y L}\right)}{d_{\alpha}\left(K_{y L}, K_{B L}^{q}\right)}=\frac{d_{\alpha}\left(K_{A L}^{p}, K_{x L}\right)}{d_{\alpha}\left(K_{x L}, K_{A L}^{q}\right)}$,

$\frac{d_{\alpha}\left(K_{B U}^{p}, K_{y U}\right)}{d_{\alpha}\left(K_{y U}, K_{B U}^{q}\right)}=\frac{d_{\alpha}\left(K_{A U}^{p}, K_{x U}\right)}{d_{\alpha}\left(K_{x U}, K_{A U}^{q}\right)}$.

In the case of polar cut based methods (see e.g. on Fig. 7 and Fig. 8):

$\forall \theta \in\left[0^{\circ}, 180^{\circ}\right]:$

fuzzinessRatio $\left(B^{p, q}, y\right)_{\theta}=\operatorname{fuzzinessRatio}\left(A^{p, q}, x\right)_{\theta}$

$\frac{d_{\theta}\left(K_{B}^{p}, K_{y}\right)}{d_{\theta}\left(K_{y}, K_{B}^{q}\right)}=\frac{d_{\theta}\left(K_{A}^{p}, K_{x}\right)}{d_{\theta}\left(K_{x}, K_{A}^{q}\right)}$.

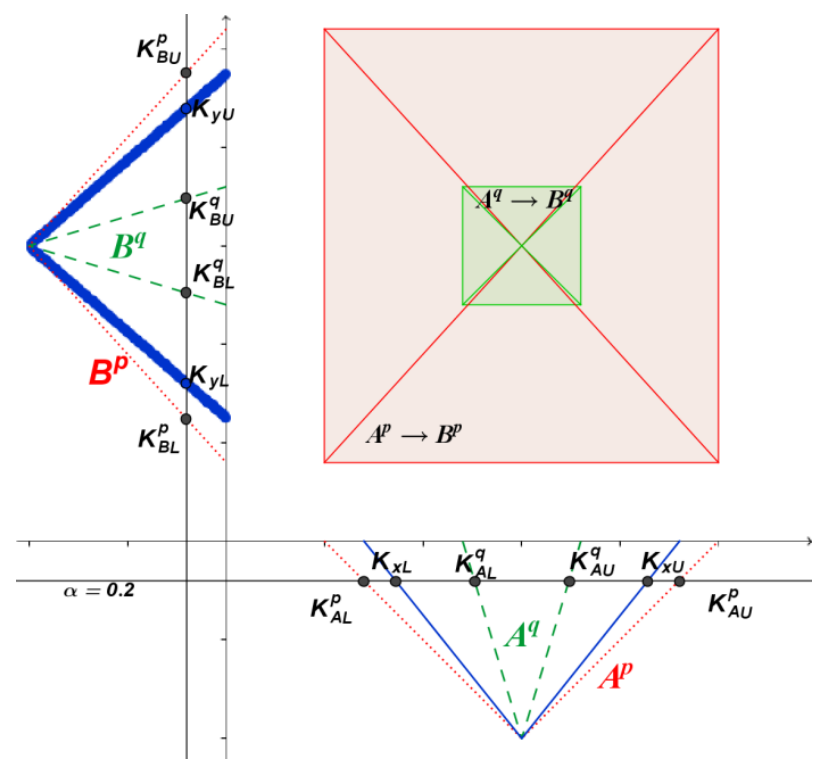

Figure 5

An example of the GDFPM $\alpha$-cut based fuzziness similarity ratio preservation reasoning step, in which the conclusion is valid, and the direction of change in fuzziness remains the same 


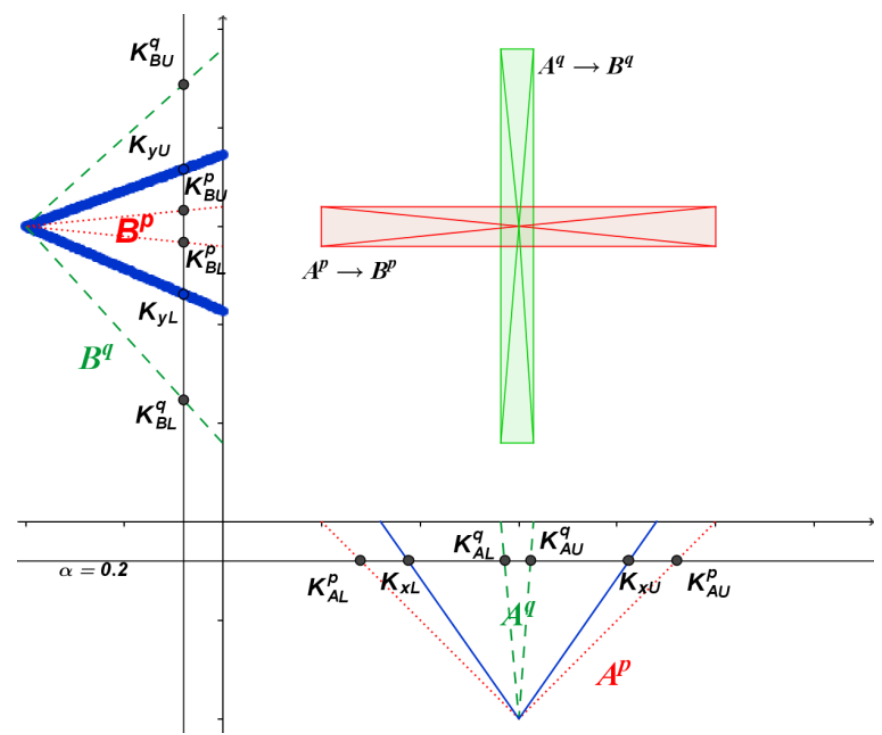

Figure 6

An example of the GDFPM $\alpha$-cut based fuzziness similarity ratio preservation reasoning step, in which the conclusion is valid, and the direction of change in fuzziness is reversed

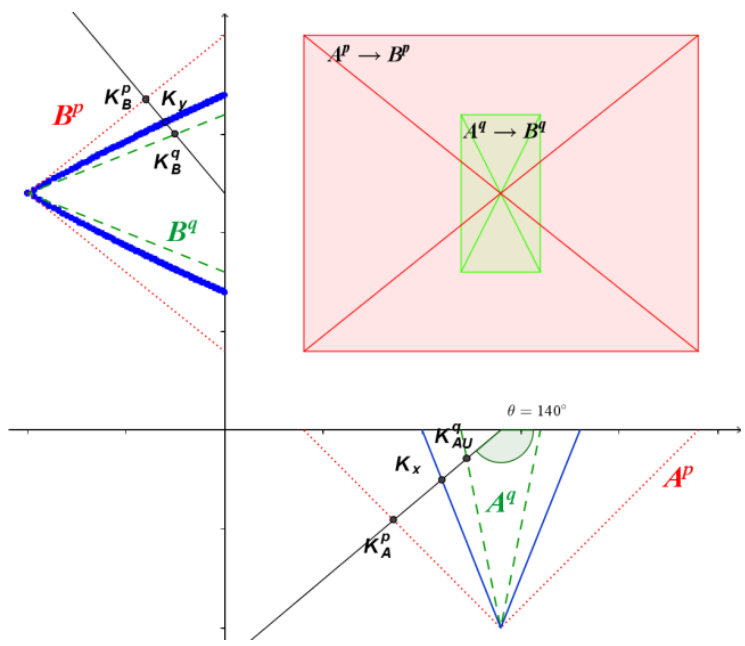

Figure 7

An example of the GDFPM polar cut based fuzziness similarity ratio preservation reasoning step, in which the conclusion is valid, and the direction of change in fuzziness remains the same 


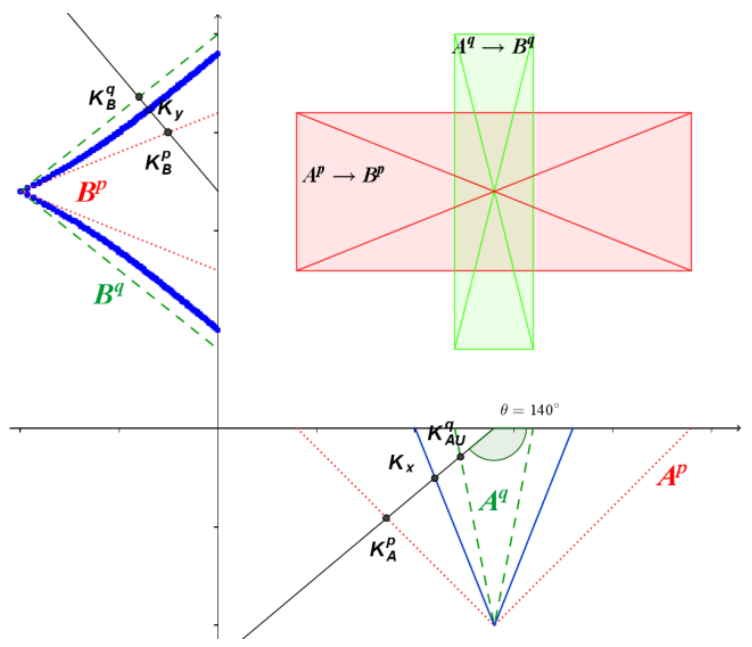

Figure 8

An example of the GDFPM polar cut based fuzziness similarity ratio preservation reasoning step, in which the conclusion is valid, and the direction of change in fuzziness is reversed

\subsection{Validity and Shape of the Conclusion}

The aim of this section is to briefly check the validity and the shape of the conclusion, i.e. to check whether or not the generated conclusion is a valid fuzzy set in general, and whether or not it preserves the piecewise linear shape of the terms. The validity of a fuzzy set can be defined as the validity of the membership function [19]. A fuzzy set $A$ is valid if:

$\forall \alpha, \alpha_{1}<\alpha_{2} \in(0,1]: \inf \left\{[A]_{\alpha}\right\} \leq \sup \left\{[A]_{\alpha}\right\}$ and

$\inf \left\{[A]_{\alpha_{1}}\right\} \leq \inf \left\{[A]_{\alpha_{2}}\right\}$ and $\sup \left\{[A]_{\alpha_{2}}\right\} \leq \sup \left\{[A]_{\alpha_{1}}\right\}$.

Remark 1 The conclusion of GDFPM is not always valid. See e.g. the example on Fig. 9.

Remark 2 GDFPM does not preserve the piecewise linear shape of the terms. See e.g. the examples on Figs. 8, 10, 11 and 12.

Remark 3 In the $\alpha$-cut based GDFPM fuzziness similarity ratio preservation reasoning step, if all fuzzy sets involved (i.e., rule antecedents, consequents and the observation) are restricted to normal triangular shaped membership functions, the conclusion will also be a valid triangular shaped fuzzy set. See e.g. the examples on Figs. 5 and 6. 


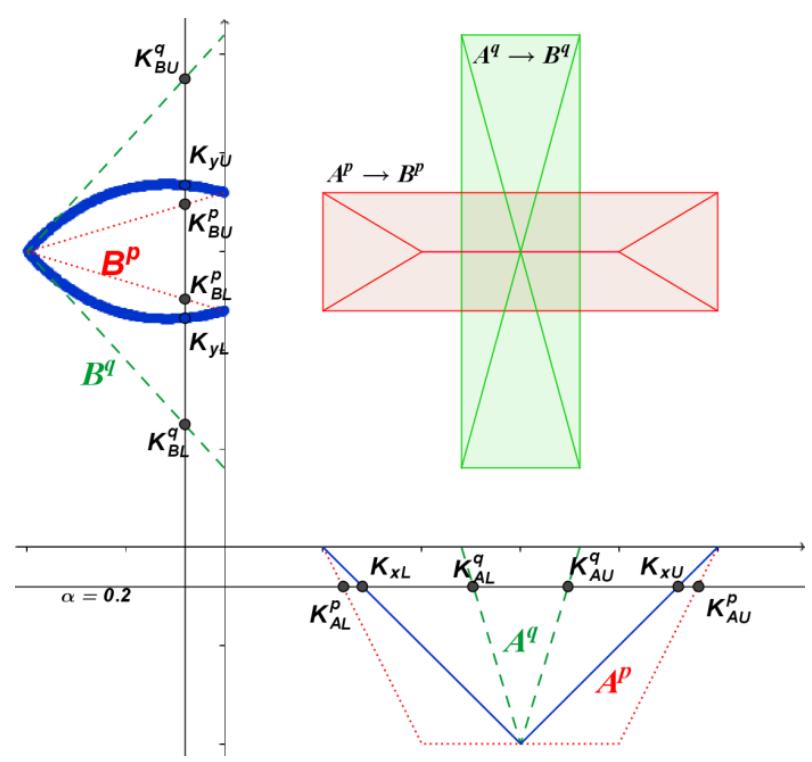

Figure 9

An example of the GDFPM $\alpha$-cut based fuzziness similarity ratio preservation reasoning step, in which the conclusion is invalid, and the direction of change in fuzziness is reversed

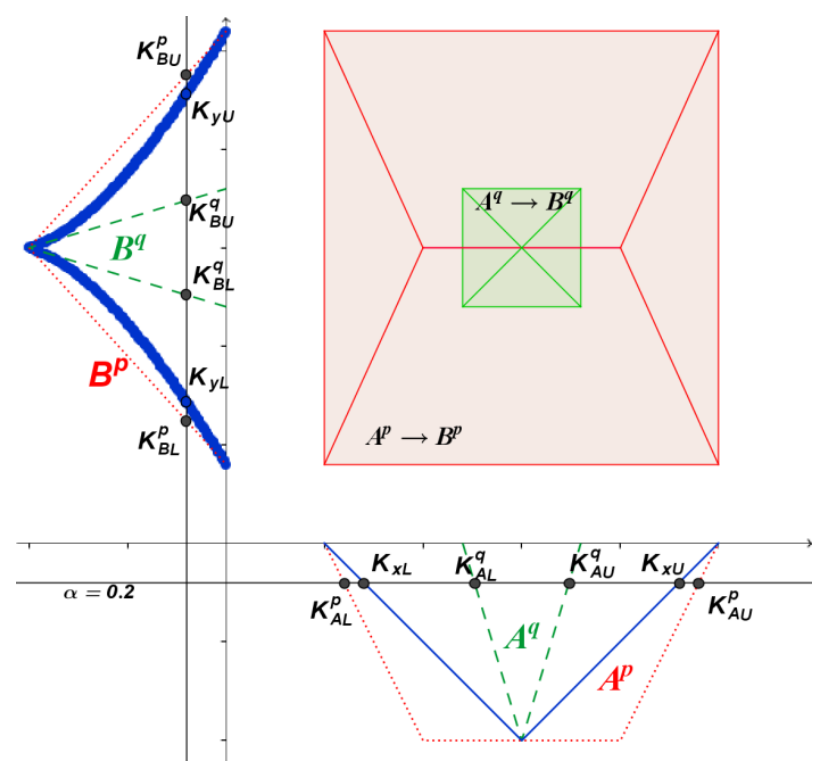

Figure 10

An example of the GDFPM $\alpha$-cut based fuzziness similarity ratio preservation reasoning step, in which the conclusion is valid, but piecewise linearity is not preserved 


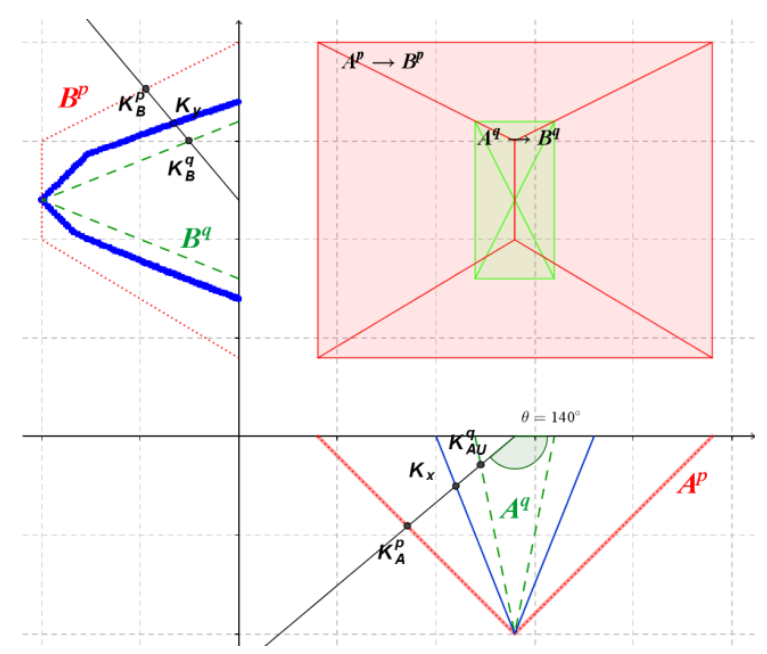

Figure 11

An example of the GDFPM polar cut based fuzziness similarity ratio preservation reasoning step, in which the direction of change in fuzziness remains the same, the conclusion is valid, and piecewise linearity is preserved

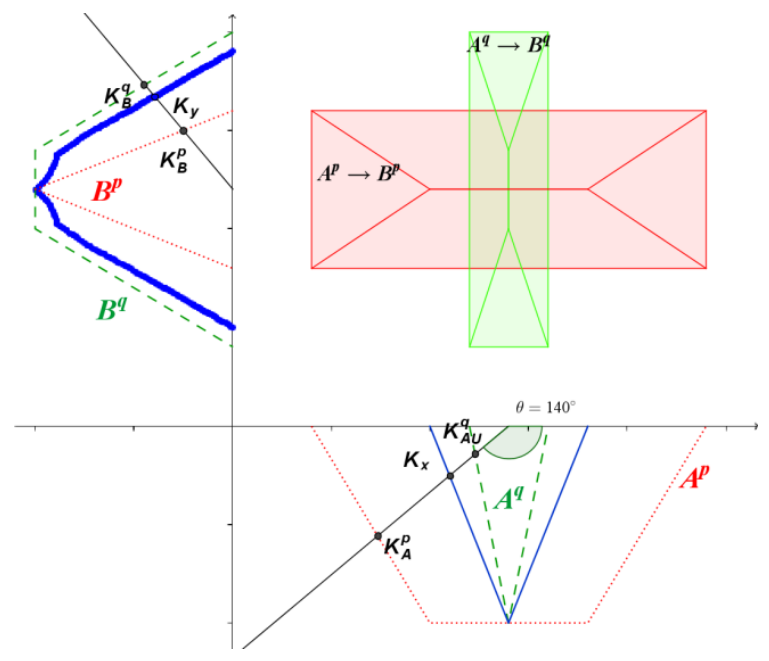

Figure 12

An example of the GDFPM polar cut based fuzziness similarity ratio preservation reasoning step, in which the direction of change in fuzziness is reversed, the conclusion is valid, but piecewise linearity is not preserved 


\section{Conclusions}

In this paper, a common "Generalized Double Fuzzy Point Methodology" (GDFPM) is introduced, which can be applied as a guideline for the double fuzzy point adaptation of any two-step FRI method. Compared to the original two-step FRI method, the first step of the proposed GDFPM approach consists of the generation of a temporal interpolated double fuzzy point rule (this is a pair of rules: one for each of the fuzzy rule sets $R^{p}$ and $R^{q}$ ) in the position of the observation. The second step of the proposed GDFPM approach consists of the determination of the conclusion based on the observation $\left(A^{*}\right)$ and the temporal interpolated double fuzzy point rule $\left(A_{i}^{p, q} \rightarrow B_{i}^{p . q}\right)$. In the latter step, GDFPM replaces the single rule reasoning step of original two-step method with a new "fuzziness similarity ratio preservation reasoning" step. As a demonstrative example, the "Least Squares Method" ("LESFRI" [9], [10]) and the "Polar a Cut" interpolation ("FRIPOC" [12]) methods were adapted to "fuzziness similarity ratio preservation reasoning" in this paper. The questions of validity of conclusion and linearity preservation in the case of the obtained two-step double fuzzy point FRI methods were also briefly examined.

\section{Acknowledgement}

This research was supported by the Hungarian National Scientific Research Fund grant no: OTKA K77809. This research was carried out as part of the TAMOP4.2.2.C-11/1/KONV-2012-0002 project with support by the European Union, cofinanced by the European Social Fund.

\section{References}

[1] L. T. Kóczy and K. Hirota, "Rule Interpolation by $\alpha$-level Sets in Fuzzy Approximate Reasoning,” BUSEFAL, Vol. 46, No. Automne, pp. 115-123, 1991

[2] P. Baranyi, D. Tikk, Y. Yam, L. T. Kóczy and L. Nádai A New Method for Avoiding Abnormal Conclusion for alpha-cut Based Rule Interpolation, $8^{\text {th }}$ IEEE International Conference on Fuzzy Systems (FUZZ-IEEE'99), Seoul, Korea, 22-25August, 1999, pp. 383-388

[3] L. T. Kóczy, K. Hirota, and T. D. Gedeon, "Fuzzy Rule Interpolation by the Conservation of Relative Fuzziness," Hirota Lab, Dept. of Comp. Intelligent and Sys. Sci., Tokyo Institute of Technology, Yokohama, Japan, Technical Report 97/2, 1997

[4] K. W. Wong, D. Tikk, T. D. Gedeon, and L. T. Kóczy, "Fuzzy Rule Interpolation for Multidimensional Input Spaces With Applications", IEEE Transactions on Fuzzy Systems, Vol. 13, No. 6, December, 2005, pp. 809819 
[5] B. Bouchon-Meunier, J. Delechamp, C. Marsala, N. Mellouli, M. Rifqi, and L. Zerrouki, "Analogy and Fuzzy Interpolation in Case of Sparse Rules," Proceedings of the EUROFUSE-SIC Joint Conference, pp. 132-136, 1999

[6] B. Bouchon-Meunier, C. Marsala, and M. Rifqi, "Interpolative Reasoning Based on Graduality," Proc. of the $9^{\text {th }}$ IEEE Int. Conf. on Fuzzy Systems (FUZZ-IEEE'00), Vol. 1, pp. 483-487, May 6-10, 2000

[7] P. Baranyi, L. T. Kóczy, and T. D. Gedeon, "A Generalized Concept for Fuzzy Rule Interpolation,” IEEE Trans. on Fuzzy Systems, Vol. 12, No. 6, pp. 820-837, December 2004

[8] Z. Huang and Q. Shen, "Fuzzy Interpolative Reasoning via Scale and Move Transformations," IEEE Trans. on Fuzzy Systems, Vol. 14, No. 2, pp. 340359, April 2006

[9] Zs. Cs. Johanyák and S. Kovács, "Fuzzy Rule Interpolation by the Least Squares Method", $7^{\text {th }}$ International Symposium of Hungarian Researchers on Computational Intelligence (HUCI 2006), November 24-25, Budapest. pp. 495-506, 2006

[10] Zs. Cs. Johanyák, "Performance Improvement of the Fuzzy Rule Interpolation Method LESFRI", in Proceeding of the $12^{\text {th }}$ IEEE International Symposium on Computational Intelligence and Informatics, Budapest, Hungary, November 21-22, 2011, pp. 271-276

[11] Zs. Cs. Johanyák, Fuzzy Rule Interpolation based on Subsethood Values, in Proceedings of 2010 IEEE Interenational Conference on Systems Man, and Cybernetics (SMC 2010), 10-13 October 2010, pp. 2387-2393

[12] Zs. Cs. Johanyák and S. Kovács, "Fuzzy Rule Interpolation Based on Polar Cuts", Advances in Soft Computing, Computational Intelligence, Theory and Applications, Bernd Reusch (Ed.), Springer Germany, pp. 499-511, 2006

[13] Zs. Cs. Johanyák, D. Tikk, S. Kovács, and K. W. Wong, "Fuzzy Rule Interpolation Matlab Toolbox - FRI Toolbox," Proc. of the IEEE World Congress on Computational Intelligence (WCCI'06), $15^{\text {th }}$ Int. Conf. on Fuzzy Systems (FUZZ-IEEE’06), pp. 1427-1433, July 16-21, 2006

[14] Zs. Cs. Johanyák, "Sparse Fuzzy Model Identification Matlab Toolbox Rulemaker Toolbox," Proceedings of IEEE $6^{\text {th }}$ International Conference on Computational Cybernetics ICCC, pp. 69-74, 2008

[15] Zs. Johanyák. Fuzzy Rule Interpolation Matlab Toolbox Website. [Online]. Available: http://fri.gamf.hu

[16] R.-E. Precup, S. Preitl and P. Korondi, "Fuzzy Controllers with Maximum Sensitivity for Servosystems", IEEE Transactions on Industrial Electronics, Vol. 54, No. 3, 2007, pp. 1298-1310 
[17] I. Perfilieva, et al., "Interpolation of Fuzzy Data: Analytical Approach and Overview", Fuzzy Sets and Systems, 2010, doi:10.1016/j.fss.2010.08.005

[18] S. Jenei, "Interpolating and Extrapolating Fuzzy Quantities Revisited - an Axiomatic Approach", Soft Comput., Vol. 5., 2001, pp. 179-193

[19] D. Tikk, Zs. Cs. Johanyák, Sz. Kovács, K. W. Wong, "Fuzzy Rule Interpolation and Extrapolation Techniques: Criteria and Evaluation Guidelines", Journal of Advanced Computational Intelligence and Intelligent Informatics, Vol. 15, No. 3, 2011, pp. 254-263

[20] S. Kovács, "Extending the Concept of Fuzzy Rule Interpolation with the Interpolation of the Fuzziness," Proceedings of the WCCI 2012 IEEE World Congress on Computational Intelligence, pp. 1106-1113, June 2012 